\title{
Inflammation and the Alteration of B-Cell Physiology in Aging
}

\author{
Magdalena Hagen Emmanuel Derudder \\ Institute for Biomedical Aging Research, University Innsbruck, Innsbruck, Austria
}

\section{Keywords}

B cells · Development · Function · Aging · Inflammation

\begin{abstract}
Aging results for the immune system in a departure from the optimal homeostatic state seen in young organisms. This divergence regrettably contributes to a higher frequency of compromised responses to infections and inefficient classical vaccination in aged populations. In B cells, the cornerstone of humoral immunity, the development and distribution of the various mature B cell subsets are impacted by aging in both humans and mice. In addition, aged mature $B$ cells demonstrate limited capacity to mount efficient antibody responses. An expected culprit for the decline in effective immunity is the rise of the systemic levels of pro-inflammatory molecules during aging, establishing a chronic low-grade inflammation. Indeed, numerous alterations affecting directly or indirectly B cells in old people and mice are reminiscent of various effects of acute inflammation on this cell type in young adults. The present mini-review will highlight the possible adverse contributions of the persistent low-level inflammation observed in susceptible older organisms to the inadequate B-cell physiology.
\end{abstract}

(c) 2019 S. Karger AG, Basel

\section{KARGER}

(C) 2019 S. Karger AG, Basel

karger@karger.com

www.karger.com/ger

\section{Introduction}

The aging of organisms is characterized by the remodelling of their constituting tissues. As a result, suboptimal physiological functions can be observed in many individuals among aged populations. The pace of decline results from the interplay between environmental factors, body internal conditions and the expression of the genome [13 ], causing the simultaneous presence of frail septuagenarians and healthy centenarians in the human elderly.

The immune system contributes to the body's homeostasis by containing or eliminating threats to an organism's integrity. Unfortunately, its proficiency in fending off pathogens and mounting optimal responses to classical vaccination/immunization is less effective with age in an increasing fraction of elderly and mice. These changes are the consequences of altered development and functionality of diverse immune cells $[4,5]$. Thus, the generation of the various mature $B$ cell populations in aging and their ability to efficiently contribute to fighting off infections is affected in older organisms $[4,6]$. Mature B cells lie at the heart of the humoral immune response, being the unique source of antibody production upon terminal differentiation [7]. Remarkably, the deterioration of immunity parallels an observed increase in systemic levels of pro-inflammatory molecules in the elderly $[3,4,8]$. Inflammation is a beneficial physiological response to stress situations in its acute form [9]. In contrast, excessive or chronic inflammatory reactions are typically detrimental

Emmanuel Derudder

Institute for Biomedical Aging Research

University Innsbruck, Rennweg 10

AT-6020 Innsbruck (Austria)

E-Mail Emmanuel.Derudder@uibk.ac.at 
to an organism, contributing to septic shock as well as to the emergence of various pathologies, such as age-related atherosclerosis and cancers [3, 8-10]. In this mini review, we focus on the basic relationship between aging, proinflammatory signals and B cells in mice and humans. The proposed adverse effects of persistent inflammation on aged B-cell physiology at the cellular level, and on some notable molecular aspects, will be especially discussed in the mouse model.

\section{Optimal B-Cell Development and Function}

The alterations of the B-cell compartment in aging have been evaluated by contrast to B-cell physiology in (young) adults. Overall, B-cell generation and function demonstrate large similarities between young mice and humans [7, 11-14]. In the more detailed mouse context, $\mathrm{B}$ cells arise from uncommitted progenitors nested in the bone marrow [12]. Ample numbers of progenitors gradually restrict their potential from acquiring diverse cell fates, following a sequence of differentiation including hematopoietic stem cells (HSCs), multi-potent progenitors and common lymphoid progenitors (CLPs) to solely commit to the B-cell lineage. This latter fate is fully enforced upon the expression of a feed forward loop involving the transcription factors E2A, EBF1 and Pax5 [12].

Within the B-cell lineage, cells undergo additional developmental steps to ultimately generate mature B cells. The hallmark of B-cell development is the rearrangement of the variable regions in the immunoglobulin (Ig) heavy $(\mathrm{H})$ and light $(\mathrm{L})$ chain loci [13]. The stochastic assembly of DNA elements, that is, $\mathrm{V}$ (variable), D (diversity) and J (joining), with the random addition of extra nucleotides, is initiated in the IgH locus at the time of commitment to the $\mathrm{B}$-cell lineage and is completed at the pro- $\mathrm{B}$ cell stage. These recombination events are mediated through the activity of the recombination-activating gene 1 and 2 proteins (RAGs) which produces targeted DNA doublestrand breaks. Subsequently, the generated $\operatorname{IgH}$ chain pairs with a surrogate light chain (SLC), composed of $\lambda 5$ and VpreB subunits, in a pre-B cell antigen receptor (BCR) expressed by pre-B cells. Pro- and pre-B cells also express the receptor for the cytokine IL-7 [13]. The above 2 receptors regulate the survival and proliferation of the $\mathrm{B}$-cell progenitors. In addition, pre-BCR- and IL-7R-signalling favour or limit, respectively, further differentiation of $B$ cells by controlling the rearrangement of the IgL locus. The decision is influenced by the proximity of the B-cell progenitors to cells secreting IL-7 [13]. Of note, IL-7 sig- nals appear not essential for B-cell development in humans, contrasting with the mouse situation [13]. Upon RAG-mediated V-to-J rearrangement in the IgL locus, the newly generated Ig light chain associates with the $\operatorname{IgH}$ chain forming a BCR expressed on immature B cells. Each $B$ cell bears a BCR with a unique specificity for antigens. The last steps of B-cell development occur in the spleen and result in the generation of a mature B-cell compartment comprising an abundant variety of BCR specificities with limited auto-reactivity. Mature B cells come in 3 main flavours, follicular (Fo), marginal zone (MZ) and B1 $B$ cells [11]. MZ B cells are the first responders to bloodborne infectious agents and $\mathrm{B} 1 \mathrm{~B}$ cells are prominent contributors to the production of natural antibodies. Fo B cells constitute the largest mature B-cell population and are major players in $\mathrm{T}$ cell-dependent responses.

Upon antigen encounter, mature B cells can enter a complex multicellular reaction, the germinal centre (GC) [7]. Interplay between B cells, stromal follicular dendritic cells (FDC) and $\mathrm{CD} 4{ }^{+} \mathrm{T}$ cells modulates the $\mathrm{B}$-cell proliferative response, selection for increased BCR affinity and class switch recombination (CSR). BCR affinity maturation depends on the accumulation of mutations in the Ig locus variable regions and CSR permits to adapt the type of IgH to the ongoing immune responses for proper antibody effector function. The latter processes are initiated by the enzyme Activation-Induced cytidine Deaminase (AID). The outcome of the GC reaction is the generation of memory B cells and antibody-secreting cells, that is, plasma cells (PCs).

\section{Chronic Inflammation and Aging}

Acute inflammation helps to cope with challenges, contributing to the removal of threats and the repair of the ensuing tissue damages [9]. Various foreign (e.g., the bacterial wall component LPS and allergens) and cell-intrinsic (e.g., ATP and HMGB1) stimuli can trigger acute inflammation. A series of mediators, such as chemokines and cytokines, is subsequently released by activated cells, like macrophages. This notably leads to the local recruitment of a variety of immune cells, swelling and/or fever. In contrast, a constant disturbance in tissue homeostasis favours the establishment of chronic inflammation, a process linked to various human pathologies, such as obesity or asthma [9]. Aging appears to resemble the latter situation, since the elderly show persistently higher systemic titres of pro-inflammatory molecules, including IL-1 $\beta$, IL- 6 and TNF $\alpha$, than younger individuals $[1,3,5$, 
8]. The constant low-grade inflammatory state parallels the increased incidence of several diseases, like diabetes, in elderly $[3,8]$. This form of inflammation is often referred to as inflammaging $[1,2]$, although the basic aspects of the concept are still debated [5]. However, not all the elderly demonstrate susceptibility to this chronic inflammation. Healthy centenarians seem to effectively counteract inflammation by increased expression of antiinflammatory molecules $[1,8]$.

The establishment of chronic inflammation during aging is evidently multifactorial [3]. Thus, genetic polymorphisms in regulatory elements have been associated with alterations of the expression of pro-inflammatory genes in old people, as in the case of the cytokine IL-6 [3]. Senescent cells, known to be mitotically incompetent but metabolically functional, accumulate with age [8]. Such cells, including human and mouse fibroblasts, have the capacity to constitutively secrete pro-inflammatory cytokines. In human, the terminally differentiated $\mathrm{CD} 8^{+}$effector $\mathrm{T}$ cells have a propensity to produce IFN- $\gamma$ and $\mathrm{TNFa}$ [4]. The number of these cells increases with age as a result of past encounters with pathogens and their accumulation is exacerbated by chronic infections, like CMV in humans [4]. The permeability of the intestinal barrier loosens with age allowing micro-organisms to enter the body and participate in the inflammatory response in old mice and humans $[3,15]$. Another contributing factor involves the cross-talk between adipocytes and immune cells resulting in the accumulation of pro-inflammatory T cells and macrophages expressing IL-6, IFN- $\gamma$ and TNFa. Such a situation is more pronounced in obese individuals [3].

\section{Bone Marrow B-Cell Development in Aging and Inflammation}

Overall, aging disturbs B-cell development in the mouse bone marrow $[6,16,17]$. The murine pre- $\mathrm{B}$ compartment appears to be the most affected, but reduced pro- and immature B cellularity have also been reported [17]. Strikingly, aging seems to introduce a high mouseto-mouse variability in early progenitor B cellularity compared to young mice. Impaired B-cell development occurs as a result of affected RAG and SLC expression, which alter pre-BCR and BCR formation, as well as decreased sensitivity to IL-7 signals [6, 16-18]. The in-depth situation in humans remains to be established. Nevertheless, available studies suggest that the amount of B cells decreases, although proportions of progenitor and ma- ture B subpopulations may not be substantially changed in the aged bone marrow $[19,20]$.

Various clues point at a role for inflammation in the altered B-cell development in aging, albeit the data is generally based on similarities with acute inflammatory responses. Indeed, pro-inflammatory senescent cells as well as terminally differentiated $\mathrm{CD}^{+}$effector $\mathrm{T}$ cells accumulate in the bones of old mice and humans respectively $[21,22]$. Correspondingly, concentrations of pro-inflammatory molecules or their production by cells are increased in bone marrow during aging [21, 23]. The balance between the negating effects of anti-inflammatory cytokines and the intensity, as well as variety, of pro-inflammatory molecules expressed could contribute to the observed variability in the deterioration of early B-cell development in susceptible aged organisms.

Inflammation can affect the differentiation of multilineage hematopoietic progenitors. Thus, aged mouse and human HSC physiology is altered and the output of this compartment reveals a bias against the production of lymphocytes due to the accumulation of stem cells with a propensity to differentiate into myeloid cells $[6,24]$. In mice, the effects of pro-inflammatory signals on young HSCs recapitulate the features of this compartment in aging $[25,26]$. Thus, LPS, including low-grade chronic treatments, promotes the differentiation of the latter cell population towards the myeloid lineage, limiting the generation of CLPs and hence B cells. LPS also induces the expression of a pro-myeloid genetic programme in aged HSCs [26]. HSC progeny cells can also be impacted. Indeed, adipocyte-induced IL- $1 \alpha$ - and IL- $1 \beta$-mediated inflammatory responses can redirect the murine multi-potent progenitor compartment towards producing myeloid progenitors to the detriment of entering the $\mathrm{B}$-cell lineage [27].

Persistent inflammation in aging could also compromise the differentiation of $\mathrm{B}$ lineage-restricted progenitor cells. A major effect of aging is the repression in B cells of the expression and/or activity of the transcription factors E2A and EBF1, which control the RAG and SLC genes, and modulate the accessibility of the Ig chain loci $[12,13$, 17]. Chronic low doses of LPS lead to reduced E2A expression in pro-B cells in mice [25]. In the elderly, pre-B cells show higher transcript levels of the E2A inhibitor $\mathrm{Id} 2$, correlating with altered accessibility of the IgH loci [19]. Id 2 expression can be stimulated by the pro-inflammatory cytokine IL-1 $\beta$ in mouse lymphocytes [28]. Interestingly, TNFa signals appear to repress EBF1 expression in human $\mathrm{CD}_{3} 4^{+}$hematopoietic progenitors and could thus contribute to the lower EBF1 levels in aged progeni- 
tor B cells [29]. Pro-B cells, as well as CLPs, from old mice are less susceptible to IL-7 stimulation, although these cells display surface levels of IL-7R similar to their counterparts in young animals [18]. In this context, TNFa and IL-1 $\beta$ signalling hinder IL-7-mediated expansion/generation of B-cell progenitors [17, 27]. IFN- $\gamma$ could also participate in the desensitization of B-cell progenitors, since its effects on cells can interfere with IL-7R signals [30]. The decreased fitness due to inflammation especially sensitizes progenitor B cells expressing high levels of preBCR to cell death in mice [17].

\section{Bone Marrow B-Cell Niches in Aging and Inflammation}

The development of hematopoietic cells requires an interplay with their cellular surroundings, such as osteoblasts and mesenchymal progenitor cells, in the so-called niches [31,32]. Niche cells modulate the development of multi-lineage hematopoietic as well as B-cell progenitors through cell-cell interactions and the secretion of soluble factors. The contribution of the various cell-types to the niche function is still debated. Nevertheless, 2 molecules, the chemokine CXCL12/SDF-1 and the growth factor IL7 , appear to be major regulators of B-cell generation in mice $[31,32]$. It was recently proposed that mesenchymal progenitor cells attract pro-B cells in a CXCL12-dependent manner to the IL-7 producing niche favouring B-cell progenitor survival and proliferation [32]. Pre-BCR expression would release $B$ cells from the effects of the niche, allowing pre-B cells to migrate away from IL-7 and further differentiate.

Aging could interfere with the support provided by the environment, notably limiting the availability of CXCL12 and/or IL-7 during B-cell generation in the bone marrow. Stromal cells from aged mice are less efficient in sustaining B-cell growth in vitro [6]. Aged mesenchymal progenitor cells express lower levels of molecules helping the development of hematopoietic cells compared with their counterparts from younger mice [22]. This reduced production seems to stem from the senescent phenotype of these cells and could be mediated by pro-inflammatory autocrine signals. Remarkably, inhibiting mesenchymal progenitor cell senescence correlates with improved expression of CXCL12 and IL-7 [22]. Stromal cell behaviour may be influenced by cells like terminally differentiated $\mathrm{CD}^{+}$effector $\mathrm{T}$ cells, which produce pro-inflammatory molecules and demonstrate increased frequency in older people [4]. Several inflammatory signals appear to affect the expression of CXCL12 and IL-7. The systemic levels of CXCL12 in the bone marrow of adult mice are negatively impacted in the presence of inflammatory mediators, such as TNFa in combination or not with IL- $1 \beta[33$, 34]. Interestingly, permanent $\mathrm{TNF} \alpha$ stimulation in vivo or treatment with this cytokine in vitro leads to lower expression of CXCL12 by bone marrow stromal cells [34]. TGF- $\beta$, which has both pro- and anti-inflammatory properties, can be found at increased levels in the blood of the elderly [3]. This cytokine represses the production of CXCL12 and IL-7 by mouse and human bone marrow stromal cells in vitro respectively [34, 35]. In mice, G-CSF treatment or sepsis-induced G-CSF expression results in the lower expression of CXCL12 and IL-7 by mesenchymal progenitor cells and osteoblasts [31]. G-CSF also leads to decreased bone cellularity of osteoblasts [31].

Reduced CXCL12 also leads to the efflux of mouse progenitor $\mathrm{B}$ cells from the bone marrow during acute inflammation [33]. However, whether such a mechanism could decrease B-cell production in the bone marrow during aging remains to be clarified.

\section{Reshaping of the Mature B-Cell Compartment in Aging}

Despite the alteration of the B-cell compartment in the bone marrow, the cellularity of mature $B$ cells in the spleen is comparable between aged and young mice [6, 16]. However, this apparent stability masks underlying disparities in the distribution of mature B-cell subsets [6, 16]. The situation in humans appears more difficult to appreciate, since most studies performed analyses based on blood samples, which have an inherent variability and may not reflect mature B-cell representation within tissues [6]. In addition, different strategies were employed to evaluate the B-cell compartment. Altogether, the B-cell fraction in the blood of elderly people appears decreased and the proportions of naive and memory subsets altered $[4,6,10]$. Similar to the bone marrow, various inflammatory molecules could influence the distribution of mature B-cell populations.

The splenic MZ B-cell population has been independently reported as being either expanded or reduced in aged mice $[16,36]$. While this variability could be caused by the genetic background of the mouse strains used in these studies [36], it could also result from disparities in inflammation levels in the animal colonies. Indeed, sustained low systemic levels of IFN- $\gamma$ were associated with decreased proportions of MZ B cells [37]. 
A striking alteration of the mouse mature B-cell compartment during aging is the accumulation of age-associated $B$ cells $(A B C)$ to the detriment of naivve Fo $B$ cells [38-41]. In agreement, Fo B cellularity decreases in the spleens and visceral adipose tissues of aged mice. Proportions of $\mathrm{ABC}$ cells also rise within peripheral blood $\mathrm{B}$ cells in women over 60 years old with rheumatoid arthritis [38]. ABC cells can be characterized by the expression of the transcription factor T-Bet as well as the surface markers $\mathrm{CD} 11 \mathrm{~b}$ and $\mathrm{CD} 11 \mathrm{c}[38,39]$. Interestingly, the generation of these cells depends on pro-inflammatory signals. Stimulation of toll-like receptors (TLRs), especially TLR7, by pro-inflammatory pathogen- or damage-associated molecular patterns (e.g., bacterial products and nucleic acids) contributes to the formation of $\mathrm{ABC}$ cells in mice [38-40]. Cytokines, such as IL-21 and IFN- $\gamma$, are also necessary [40]. While T cells are obvious candidates to secrete the latter 2 molecules, it has been shown that adipocytes are also capable of producing these cytokines in mice [41]. Consistent, adipocytes effectively promote the differentiation of Fo B into ABC cells. In addition to ABC cells, an IgD and CD27-double negative (DN) memory $\mathrm{B}$-cell population is more often detected in elderly than young people [10]. DN B cells also expand in patients with autoimmunity, suggesting that their presence in old individuals could result from chronic inflammation.

The frequency of the third major mature B-cell subset in mice, the B1 B cells, appears to rise with age in their site of predilection, the peritoneal cavity, but also in the spleen $[6,42]$. This increase could involve not only extended lifespan or the known self-renewal potential of mature B1 B cells, but may also result from unscathed development of their bone marrow progenitors during aging [42]. Indeed, $\mathrm{B} 1$ precursor cells display a reduced expression of the pre-BCR constituent $\lambda 5$ in the bone marrow and are thus protected from TNFa-mediated elimination of $\mathrm{B}$ cells with high levels of pre-BCR, as discussed earlier [42].

\section{Modulation of B-Cell Function by Inflammatory Signals}

Elderly people and aged mice can demonstrate increased susceptibility to infections and a limited response to classical vaccination/immunization $[4,6]$. While these age-mediated defects are likely multifactorial, a key contribution is impaired GC reactions. Sub-optimal GC reactions could result from the impact of inflammation on cells participating in the process, that is, $\mathrm{FDCs}, \mathrm{CD} 4^{+} \mathrm{T}$ cells and GC B cells.

B Cells in Aging
FDCs from old mice have a deteriorated capacity to support B-cell antibody production [43]. This could partly be due to increased susceptibility to Fas-mediated cell death. Indeed, TNFa promotes the expression of the proapoptotic Fas receptor by a human FDC cell line [44].

$\mathrm{CD} 4^{+} \mathrm{T}$ follicular helper $\left(\mathrm{T}_{\mathrm{FH}}\right)$ cells assist, while $\mathrm{CD} 4^{+}$ $\mathrm{T}$ follicular regulatory $\left(\mathrm{T}_{\mathrm{FR}}\right)$ cells suppress the maturation of GC B cells, like differentiation into PCs. Consistent with an impaired GC reaction, immunization of old mice results in a $\mathrm{T}_{\mathrm{FR}} / \mathrm{T}_{\mathrm{FH}}$ ratio in favour of $\mathrm{T}_{\mathrm{FR}}$ cells [45]. In addition, $\mathrm{T}_{\mathrm{FH}}$ cell function is compromised. However, a role for inflammation has not yet been clearly established. STAT3, a transcription factor activated by IL-6, contributes to the appearance of both $\mathrm{T}_{\mathrm{FH}}$ and $\mathrm{T}_{\mathrm{FR}}$ cells. Nevertheless, the differentiation of $\mathrm{T}_{\mathrm{FR}}$ cells seems more dependent on STAT3 activity [46]. Thus, the generation of $\mathrm{T}_{\mathrm{FR}}$ may be advantaged under inflammatory conditions during aging.

Inflammation can also interfere with the B-cell response to stimulation. TNFa affects mouse splenic B-cell activation, reducing the production of antibodies in vitro [36]. Notably, B cells pre-treated with TNFa show decreased expression of E2A and AID, 2 endogenous regulators of GC B-cell physiology, upon stimulation [36]. The lower synthesis of the latter 2 molecules is (partly) achieved via the repressive action of microRNAs. Aged B cells show higher levels of the pro-inflammatory miR-155 as well as miR-16, which target AID and E2A, respectively [47]. Strikingly, inhibiting TNFa activity improves AID expression and CSR in activated aged mouse B cells [36]. Long-term production of antibodies is achieved by PCs that have homed in a CXCL12-dependent manner to survival niches in the bone marrow [20]. As mentioned [33], inflammation leads to an impaired production of CXCL12 in the bone marrow, which may limit the recruitment of newly generated PCs and contribute to their reduced accumulation in the bone marrow of elderly people and old mice $[20,48]$.

\section{Effects of Inflammation and Aging on the B-Cell Repertoire}

B cells from old people and aged mice show a substantial heterogeneity in the composition of their Ig variable regions, but not the elderly with poor health [49-51]. Thus, the aged B-cell repertoire is not typically dominated by a restricted number of B-cell clones, except in frail individuals in which (pre-)malignant B cells may have expanded. However, increased levels of auto-antibodies and 

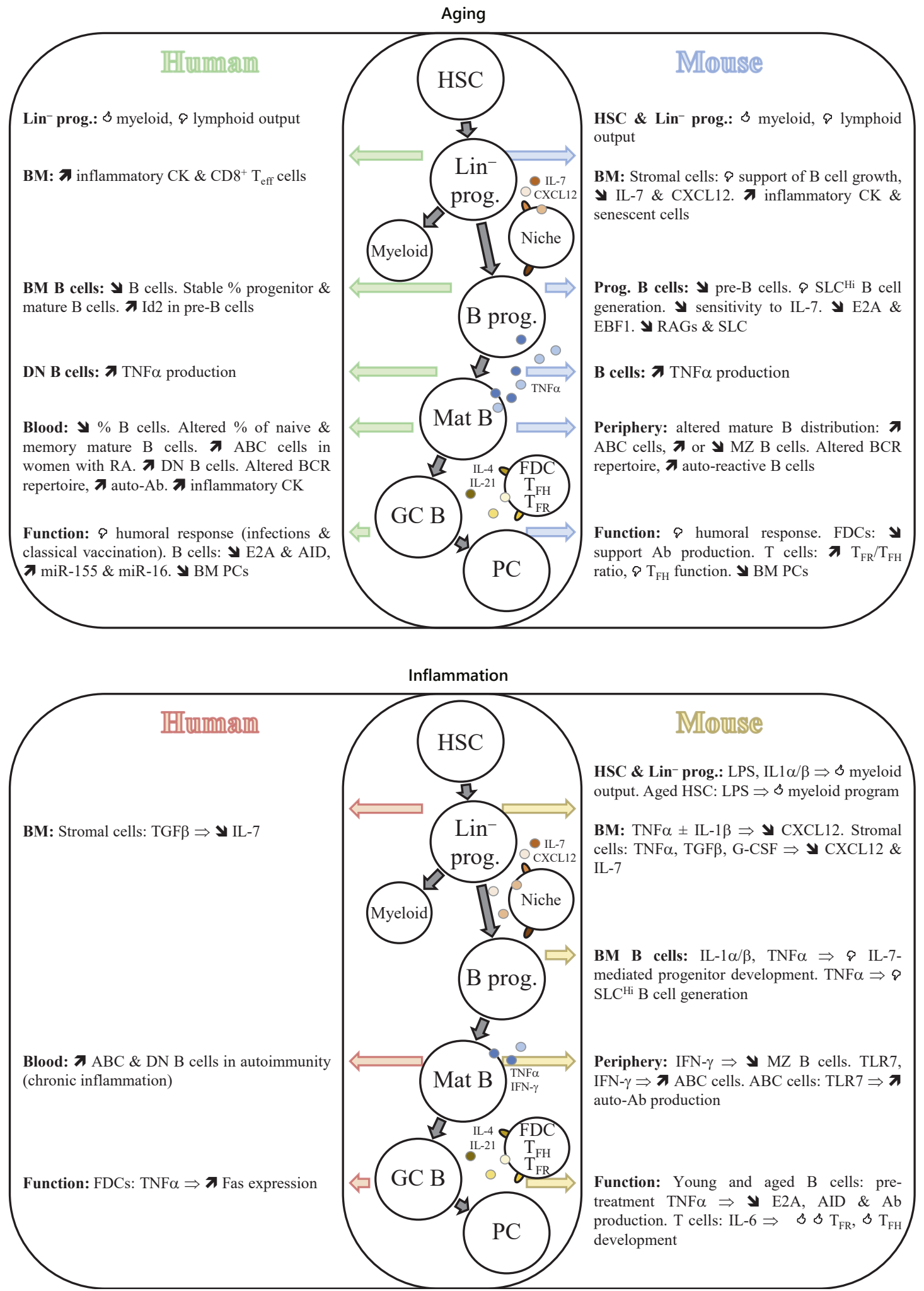

1

(For legend see next page.) 
a greater mutation load in Ig genes can be seen in the elderly population $[6,10,16]$, indicating more subtle alterations of the repertoire. While $B$ cells against the bacterial- and self-antigen phosphorylcholine are present in old mice, proportions of clones with BCR reactivity useful against infection are decreased [52].

As mentioned, the generation of mouse $\mathrm{B}$-cell progenitors expressing high levels of pre-BCR is affected by inflammation. Interestingly, the ratio between protective versus non-protective specificities against phosphorylcholine is in favour of the latter in the repertoire of old mice with B cells deriving mainly from low SLC-expressing progenitors [52]. In support of a role for the pre-BCR in the selection of B cells, mice deficient for the SLC show an accumulation of auto-reactive $B$ cells, apparently of the ABC cell fate [53]. Pro-inflammatory TLR7 signalling is necessary for the accumulation of, and antibody production by, auto-reactive $A B C$ cells, in mice [38]. Of note, increased proportions of $\mathrm{ABC}$ as well as $\mathrm{DN} B$ cells have been associated with auto-immune diseases in humans $[10,38]$. Thus, the expansion of auto-reactive B-cell populations among elderly could be a consequence of chronic inflammation. (Self-)antigen-experienced B cells may also contribute to shaping the repertoire by filling up the mature compartment, excluding naive B cells, as observed in Ig transgenic mice [54].

\section{Contribution of B Cells to Inflammation in Aging}

Aged mouse B cells, including pro-B as well as mature $B$ cells, express higher levels of TNFa than their younger counterparts at steady-state $[36,55]$. DN B cells from old people also express more pro-inflammatory molecules compared to other mature B subsets [56]. These observations indicate that $\mathrm{B}$ cells could participate in their own demise. In vitro, $A B C$ cells from aged mice secrete sufficient TNFa to impair the generation of young pro-B cells, suggesting a possible contribution of bone marrow resident $\mathrm{ABC}$ cells to altered B-cell development [17]. In addition, mouse $\mathrm{ABC}$ cells produce along TNFa other cytokines, notably IFN- $\gamma$, which could indirectly enable damaging the aged B-cell compartment $[30,39]$. Indeed, in their antigen presenting function, these cells favour $\mathrm{CD}^{+}$ $\mathrm{T}$-cell differentiation towards the pro-inflammatory $\mathrm{Th}_{17}$ cell fate [39].
Fig. 1. Effects of aging and inflammation on the generation and function of B cells. Overview of remarkable changes in B-cell development and function during aging (top) in the human (left) and mouse (right) context. In the BM, the production of myeloid cells by hematopoietic stem and uncommitted progenitor cells (HSC; $\mathrm{Lin}^{-}$prog.) is favoured (thumbs-up symbol), while the generation of lymphocytes is impaired (thumbs-down symbol). Aging also affects directly B-cell progenitors (B prog.), resulting in a decreased (arrow pointing down) amount of BM B cells in humans and a reduced cellularity of mouse pre-B cells, notably due to the restricted maturation of progenitors expressing high levels of SLC and the decreased sensitivity to IL-7 signals. These changes are linked to the altered expression of modulators of B-cell development (Id2, E2A, EBF1, RAGs and SLC). In addition, BM niches, like stromal cells, promote less efficiently B-cell growth, correlating with a reduced production of IL-7 and CXCL12. The aged mature B (Mat B)-cell compartment and B-cell repertoire are also remodelled. While the impact on MZ B cells remains to be clarified, ABC and DN B cells, which contribute to the increase (arrow pointing up) in auto-Ab titers and auto-reactive B cells, accumulate substantially. Finally, the GC reaction is impaired in old organisms as well. Activated B cells express lower levels of E2A and AID, which parallels the increased levels of their repressors miR-155 and miR-16. The humoral response is also affected by decreased support of FDC to $\mathrm{Ab}$ production, favoured generation of follicular regulatory $\mathrm{T}$ cells $\left(\mathrm{T}_{\mathrm{FR}}\right)$, compromised follicular helper $\mathrm{T}\left(\mathrm{T}_{\mathrm{FH}}\right)$ cell function and lower numbers of long-lived PCs in BM. A role for inflamma- tion in aging is consistent with the accumulation of proinflammatory senescent stromal as well as terminally differentiated $\mathrm{T}$ effector $\mathrm{CD}^{+}$cells in old $\mathrm{BM}$, and higher levels and/or production of pro-inflammatory cytokines (CK). Aged B cells also generate more TNFa. Similarities can be seen between the impact of inflammation, especially the effects of acute inflammation in young adults, on B cells (bottom) and the alterations of their development and function in aging. Inflammation leads to $(\Rightarrow)$ the preferred generation of myeloid cells by HSC and uncommitted progenitors, the expression of a myeloid program in aged HSCs, as well as reduced levels and lower production by stromal cells of Bcell growth supporting IL-7 and CXCL12. In addition, inflammation impairs IL-7-mediated generation of B-cell progenitors, especially the maturation of high SLC-expressing cells. Inflammatory signals also inhibit the formation of MZ B cells, promote the accumulation of DN B and ABC cells and stimulate the production of auto-Ab by the latter cells. Finally, inflammation interferes with the humoral response too. As a result, the expression of E2A, AID and $\mathrm{Ab}$ production by $\mathrm{B}$ cells is reduced and levels of the pro-apoptotic Fas receptor are increased on FDCs, possibly affecting their physiology. The formation of $\mathrm{T}_{\mathrm{FR}}$ cells appears also advantaged compared to $\mathrm{T}_{\mathrm{FH}}$ cells under such conditions. $\mathrm{BM}$, bone marrow; HSC, hematopoietic stem cell; FDC, follicular dendritic cells; PC, plasma cell; DN, IgD- and CD27-double negative; SLC, surrogate light chain; Ab, antibody; $\mathrm{BCR}$, B-cell antigen receptor; $\mathrm{AID}$, activation-induced cytidine deaminase; $\mathrm{ABC}$, age-associated $\mathrm{B}$ cell; GC, germinal centre; RA, rheumatoid arthritis. 


\section{Concluding Remarks}

Aging of the B-cell compartment is surely an intricate process. Similarities can nevertheless be noticed with the impacts of acute inflammation on these cells in young organisms (Fig. 1). It thus seems reasonable to consider that the chronic low-grade inflammation seen in aging causes these effects on B-cell physiology in susceptible old organisms, that is, not protected by anti-inflammatory signals. However, the levels of CXCL12 are not diminished in old mice and its expression by human mononuclear cells is relatively stable with age, in the bone marrow [21, 23]. In addition, the systemic expression of repressors of inflammation did not improve B-cell development in aged mice [55]. These findings suggest that local, rather than systemic, concentrations of cytokines/chemokines may be functionally critical. Alternatively, the age-associated inflammation may only involve a fraction of the effects of an acute inflammatory response.

While limiting systemic inflammation did not promote the development of B cells in aged mice, it ameliorated their cellular fitness [55]. A graded response of B cells to the intensity of chronic inflammation could thus be envisioned. Consistently, the transcript levels of TNFa in resting B cells from old mice negatively correlate with the induction of AID expression upon stimulation [36]. Similarly, a perturbed B-cell repertoire is observed in frail elderly, who show heightened signs of inflammation $[1-3,8,49]$.
Could interventions limit the detrimental effects of persistent inflammation on humoral immunity in susceptible elderly? A first possibility would be to inhibit TLR7 signalling using antibodies [57], with the aim to shrink the ABC cell population. Such a treatment could help reducing the production of auto-antibodies and expanding the non-antigen-experienced follicular B population [38], to generate a more naïve mature $B$ compartment mimicking the one observed in healthy people with long life-expectancy [58]. Alternatively, the level of chronic inflammation could be minimized before vaccination to improve the humoral response in the elderly. Pre-treatment of old individuals with an inhibitor of p38 MAPK, which regulates the expression of pro-inflammatory molecules, ameliorates the T-cell infiltration in the skin upon varicella zoster virus infection [59]. Finally, another approach could consist of overriding the chronic adverse inflammation by an acute beneficial one using strong pro-inflammatory adjuvants during vaccination, as supported by the efficacy of the $\mathrm{AS} \mathrm{1}_{\mathrm{B}}$ adjuvant-containing Shingrix vaccine in elderly [60].

\section{Acknowledgement}

The authors are thankful for support from the University Innsbruck Nachwuchsförderung (2017/BIO-5) and the Tiroler Wissenschaftsförderung (UNI-0404/2310). We apologize to many colleagues for not reporting all the original literature as a consequence of space constraints.

\section{References}

1 Franceschi C, Capri M, Monti D, Giunta S, Olivieri F, Sevini F, et al. Inflammaging and antiinflammaging: a systemic perspective on aging and longevity emerged from studies in humans. Mech Ageing Dev. 2007 Jan;128(1):92105.

2 Franceschi C, Garagnani P, Vitale G, Capri M, Salvioli S. Inflammaging and 'Garb-aging'. Trends Endocrinol Metab. 2017 Mar;28(3): 199-212.

3 Ferrucci L, Fabbri E. Inflammageing: chronic inflammation in ageing, cardiovascular disease, and frailty. Nat Rev Cardiol. 2018 Sep; 15(9):505-22.

4 Pinti M, Appay V, Campisi J, Frasca D, Fülöp T, Sauce D, et al. Aging of the immune system: focus on inflammation and vaccination. Eur J Immunol. 2016 Oct;46(10):2286-301.

5 Nikolich-Žugich J. The twilight of immunity: emerging concepts in aging of the immune system. Nat Immunol. 2018 Jan;19(1):10-9.

6 Kogut I, Scholz JL, Cancro MP, Cambier JC. B cell maintenance and function in aging. Semin Immunol. 2012 Oct;24(5):342-9.
7 Zhang Y, Garcia-Ibanez L, Toellner KM. Regulation of germinal center B-cell differentiation. Immunol Rev. 2016 Mar;270(1):8-19.

8 Freund A, Orjalo AV, Desprez PY, Campisi J. Inflammatory networks during cellular senescence: causes and consequences. Trends $\mathrm{Mol}$ Med. 2010 May;16(5):238-46.

9 Medzhitov R. Origin and physiological roles of inflammation. Nature. 2008 Jul;454(7203): 428-35.

10 Bulati M, Caruso C, Colonna-Romano G. From lymphopoiesis to plasma cells differentiation, the age-related modifications of B cell compartment are influenced by "inflamm-ageing”. Ageing Res Rev. 2017 Jul;36:125-36.

11 Allman D, Pillai S. Peripheral B cell subsets. Curr Opin Immunol. 2008 Apr;20(2):149-57.

12 Northrup DL, Allman D. Transcriptional regulation of early B cell development. Immunol Res. 2008;42(1-3):106-17.

13 Clark MR, Mandal M, Ochiai K, Singh H. Orchestrating B cell lymphopoiesis through interplay of IL-7 receptor and pre-B cell receptor signalling. Nat Rev Immunol. 2014 Feb;14(2): 69-80.
14 Pieper K, Grimbacher B, Eibel H. B-cell biology and development. J Allergy Clin Immunol. 2013 Apr;131(4):959-71.

15 Spychala MS, Venna VR, Jandzinski M, Doran SJ, Durgan DJ, Ganesh BP, et al. Age-related changes in the gut microbiota influence systemic inflammation and stroke outcome. Ann Neurol. 2018 Jul;84(1):23-36.

16 Miller JP, Cancro MP. B cells and aging: balancing the homeostatic equation. Exp Gerontol. 2007 May;42(5):396-9.

17 Ratliff M, Alter S, McAvoy K, Frasca D, Wright JA, Zinkel SS, et al. In aged mice, low surrogate light chain promotes pro-B-cell apoptotic resistance, compromises the PreBCR checkpoint, and favors generation of autoreactive, phosphorylcholine-specific B cells. Aging Cell. 2015 Jun;14(3):382-90.

18 Miller JP, Allman D. The decline in B lymphopoiesis in aged mice reflects loss of very early B-lineage precursors. J Immunol. 2003 Sep; 171(5):2326-30. 
19 Jensen K, Rother MB, Brusletto BS, Olstad OK, Dalsbotten Aass HC, van Zelm MC, et al. Increased ID2 levels in adult precursor B cells as compared with children is associated with impaired Ig locus contraction and decreased bone marrow output. J Immunol. 2013 Aug;191(3): $1210-9$.

20 Pritz T, Lair J, Ban M, Keller M, Weinberger B, Krismer M, et al. Plasma cell numbers decrease in bone marrow of old patients. Eur J Immunol. 2015 Mar;45(3):738-46.

21 Pangrazzi L, Meryk A, Naismith E, Koziel R, Lair J, Krismer M, et al. "Inflamm-aging" influences immune cell survival factors in human bone marrow. Eur J Immunol. 2017 Mar;47(3): 481-92.

22 Gao B, Lin X, Jing H, Fan J, Ji C, Jie Q, et al. Local delivery of tetramethylpyrazine eliminates the senescent phenotype of bone marrow mesenchymal stromal cells and creates an antiinflammatory and angiogenic environment in aging mice. Aging Cell. 2018 Jun;17(3):e12741.

23 Guidi N, Sacma M, Ständker L, Soller K, Marka $\mathrm{G}$, Eiwen K, et al. Osteopontin attenuates aging-associated phenotypes of hematopoietic stem cells. EMBO J. 2017 Apr;36(7):840-53.

24 Beerman I, Maloney WJ, Weissmann IL, Rossi DJ. Stem cells and the aging hematopoietic system. Curr Opin Immunol. 2010 Aug;22(4): 500-6.

25 Esplin BL, Shimazu T, Welner RS, Garrett KP, Nie L, Zhang Q, et al. Chronic exposure to a TLR ligand injures hematopoietic stem cells. J Immunol. 2011 May;186(9):5367-75.

26 Mann M, Mehta A, de Boer CG, Kowalczyk MS, Lee K, Haldeman P, et al. Heterogeneous Responses of Hematopoietic Stem Cells to Inflammatory Stimuli Are Altered with Age. Cell Rep. 2018 Dec;25(11):2992-3005.e5.

27 Kennedy DE, Knight KL. Inhibition of B Lymphopoiesis by Adipocytes and IL-1-Producing Myeloid-Derived Suppressor Cells. J Immunol. 2015 Sep;195(6):2666-74.

28 Hwang SM, Sharma G, Verma R, Byun S, Rudra D, Im SH. Inflammation-induced Id 2 promotes plasticity in regulatory T cells. Nat Commun. 2018 Nov;9(1):4736.

29 Smits K, De Smedt M, Naessens E, De Smet G, Stove V, Taghon T, et al. Tumor necrosis factor promotes T-cell at the expense of B-cell lymphoid development from cultured human CD34+ cord blood cells. Exp Hematol. 2007 Aug;35(8):1272-8.

30 de Bruin AM, Voermans C, Nolte MA. Impact of interferon- $\gamma$ on hematopoiesis. Blood. 2014 Oct;124(16):2479-86.

31 Day RB, Bhattacharya D, Nagasawa T, Link DC. Granulocyte colony-stimulating factor reprograms bone marrow stromal cells to actively suppress B lymphopoiesis in mice. Blood. 2015 May; 125(20):3114-7.

32 Fistonich C, Zehentmeier S, Bednarski JJ, Miao $\mathrm{R}$, Schjerven H, Sleckman BP, et al. Cell circuits between $B$ cell progenitors and IL-7+ mesenchymal progenitor cells control B cell development. J Exp Med. 2018 Oct;215(10):2586-99.

33 Ueda Y, Yang K, Foster SJ, Kondo M, Kelsoe G. Inflammation controls B lymphopoiesis by regulating chemokine CXCL12 expression. J Exp Med. 2004 Jan;199(1):47-58.

34 Zhang Q, Guo R, Schwarz EM, Boyce BF, Xing L. TNF inhibits production of stromal cell-derived factor 1 by bone stromal cells and increases osteoclast precursor mobilization from bone marrow to peripheral blood. Arthritis Res Ther. 2008;10(2):R37.

35 Tang J, Nuccie BL, Ritterman I, Liesveld JL, Abboud CN, Ryan DH. TGF-beta down-regulates stromal IL-7 secretion and inhibits proliferation of human B cell precursors. J Immunol. 1997 Jul;159(1):117-25.

36 Frasca D, Romero M, Diaz A, Alter-Wolf S, Ratliff M, Landin AM, et al. A molecular mechanism for TNF- $\alpha$-mediated downregulation of B cell responses. J Immunol. 2012 Jan;188(1): 279-86.

37 Hodge DL, Berthet C, Coppola V, Kastenmüller W, Buschman MD, Schaughency PM, et al. IFN-gamma AU-rich element removal promotes chronic IFN-gamma expression and autoimmunity in mice. J Autoimmun. 2014 Sep; 53:33-45.

38 Rubtsov AV, Rubtsova K, Fischer A, Meehan RT, Gillis JZ, Kappler JW, et al. Toll-like receptor 7 (TLR7)-driven accumulation of a novel $\mathrm{CD} 11 \mathrm{c} \otimes \mathrm{B}$-cell population is important for the development of autoimmunity. Blood. 2011 Aug;118(5):1305-15.

39 Hao Y, O’Neill P, Naradikian MS, Scholz JL, Cancro MP. A B-cell subset uniquely responsive to innate stimuli accumulates in aged mice. Blood. 2011 Aug;118(5):1294-304.

40 Naradikian MS, Myles A, Beiting DP, Roberts KJ, Dawson L, Herati RS, et al. Cutting Edge: IL-4, IL-21, and IFN- $\gamma$ Interact To Govern Tbet and CD11c Expression in TLR-Activated B Cells. J Immunol. 2016 Aug;197(4):1023-8.

41 Frasca D, Diaz A, Romero M, Vazquez T, Blomberg BB. Obesity induces pro-inflammatory B cells and impairs B cell function in old mice. Mech Ageing Dev. 2017 Mar;162:91-9.

42 Alter-Wolf S, Blomberg BB, Riley RL. Old mice retain bone marrow $\mathrm{B} 1$ progenitors, but lose B2 precursors, and exhibit altered immature $B$ cell phenotype and light chain usage. Mech Ageing Dev. 2009 Jun;130(6):401-8.

43 Aydar Y, Balogh P, Tew JG, Szakal AK. Follicular dendritic cells in aging, a "bottle-neck" in the humoral immune response. Ageing Res Rev. 2004 Jan;3(1):15-29.

44 Park SM, Park HY, Lee TH. Functional effects of TNF-alpha on a human follicular dendritic cell line: persistent NF-kappa B activation and sensitization for Fas-mediated apoptosis. J Immunol. 2003 Oct;171(8):3955-62.

45 Sage PT, Tan CL, Freeman GJ, Haigis M, Sharpe AH. Defective TFH Cell Function and Increased TFR Cells Contribute to Defective Antibody Production in Aging. Cell Rep. 2015 Jul;12(2):163-71.

$46 \mathrm{Wu}$ H, Xie MM, Liu H, Dent AL. Stat3 Is Important for Follicular Regulatory T Cell Differentiation. PLoSOne.2016May;11(5):e0155040.

47 Frasca D, Diaz A, Romero M, Ferracci F, Blomberg BB. MicroRNAs miR-155 and miR-16 Decrease AID and E47 in B Cells from Elder- ly Individuals. J Immunol. 2015 Sep;195(5): 2134-40.

48 Han S, Yang K, Ozen Z, Peng W, Marinova E, Kelsoe G, et al. Enhanced differentiation of splenic plasma cells but diminished long-lived high-affinity bone marrow plasma cells in aged mice. J Immunol. 2003 Feb;170(3):1267-73.

49 Gibson KL, Wu YC, Barnett Y, Duggan O, Vaughan R, Kondeatis E, et al. B-cell diversity decreases in old age and is correlated with poor health status. Aging Cell. 2009 Feb;8(1):18-25.

50 Martin V, Bryan Wu YC, Kipling D, DunnWalters D. Ageing of the B-cell repertoire. Philos Trans R Soc Lond B Biol Sci. 2015 Sep; 370(1676):20140237.

51 Russell Knode LM, Naradikian MS, Myles A, Scholz JL, Hao Y, Liu D, et al. Age-Associated B Cells Express a Diverse Repertoire of VH and Vк Genes with Somatic Hypermutation. J Immunol. 2017 Mar; 198(5):1921-7.

52 Khomtchouk K, Alter S, Ratliff M, Blomberg $\mathrm{BB}$, Riley RL. In old BALB/c mice, bone marrow pre- $B$ cell and surrogate light chain reduction is associated with increased B cell reactivity to phosphorylcholine, but reduced T15 idiotype dominance. Mech Ageing Dev. 2017 Mar; 162:53-62.

53 Aranburu A, Höök N, Gerasimcik N, Corleis B, Ren W, Camponeschi A, et al. Age-associated $B$ cells expanded in autoimmune mice are memory cells sharing H-CDR3-selected repertoires. Eur J Immunol. 2018 Mar;48(3):509-21.

54 Johnson SA, Rozzo SJ, Cambier JC. Aging-dependent exclusion of antigen-inexperienced cells from the peripheral B cell repertoire. J Immunol. 2002 May; 168(10):5014-23.

55 Henry CJ, Casás-Selves M, Kim J, Zaberezhnyy V, Aghili L, Daniel AE, et al. Aging-associated inflammation promotes selection for adaptive oncogenic events in B cell progenitors. J Clin Invest. 2015 Dec;125(12):4666-80.

56 Frasca D, Diaz A, Romero M, Blomberg BB. Human peripheral late/exhausted memory B cells express a senescent-associated secretory phenotype and preferentially utilize metabolic signaling pathways. Exp Gerontol. 2017 Jan;87 Pt A:113-20.

57 Kanno A, Tanimura N, Ishizaki M, Ohko K, Motoi Y, Onji M, et al. Targeting cell surface TLR7 for therapeutic intervention in autoimmune diseases. Nat Commun. 2015 Feb;6(1): 6119.

58 Colonna-Romano G, Buffa S, Bulati M, Candore $\mathrm{G}$, Lio $\mathrm{D}$, Pellicanò $\mathrm{M}$, et al. $\mathrm{B}$ cells compartment in centenarian offspring and old people. Curr Pharm Des. 2010;16(6):604-8.

59 Vukmanovic-Stejic M, Chambers ES, SuárezFariñas M, Sandhu D, Fuentes-Duculan J, Patel N, et al. Enhancement of cutaneous immunity during aging by blocking $\mathrm{p} 38$ mitogenactivated protein (MAP) kinase-induced inflammation. J Allergy Clin Immunol. 2018 Sep; 142(3):844-56.

60 Lal H, Cunningham AL, Godeaux O, Chlibek R, Diez-Domingo J, Hwang SJ, et al.; ZOE-50 Study Group. Efficacy of an adjuvanted herpes zoster subunit vaccine in older adults. N Engl J Med. 2015 May;372(22):2087-96. 